\title{
GESTIÓN DE LA CALIDAD ISO 9001 EN ALMAZARAS DEL SUR DE ESPAÑA
}

María T. Mazal, Wílmer S. Sepúlveda

Carlos E. Campo ${ }^{3}$, Alberto M. Berga

\section{Resumen}

El objetivo del presente trabajo es valorar las relaciones entre: i) el grado de cumplimiento de la norma ISO 9001 con el tamaño de la empresa y, ii) los diversos apartados de la misma, en un conjunto de almazaras ubicadas en el sur de España. La información empleada proviene de una muestra conformada por 30 almazaras ubicadas en Andalucía, que eran susceptibles de certificación. Para el análisis de la información se emplearon métodos estadísticos multivariantes. Los resultados sugieren la existencia de una fuerte relación entre el grado de cumplimiento de la norma ISO 9001 con el tamaño de las almazaras, en el sentido que las empresas más grandes eran las más susceptibles de certificación. Asimismo, se encontró una estrecha relación entre el grado de cumplimiento de diversos capítulos de la norma.

\section{Palabras clave}

ISO 9001; gestión de la calidad; almazara.

\begin{abstract}
The aim of this study is to assess the relationship between: i) the degree of compliance of ISO 9001 with the company size, and ii) the various sections of the same, a set of mills in southern Spain. The information used came from a sample composed of 30 mills in Andalusia, which were capable of certification. To measure the relationship between the degree of compliance with the rule with the size of the company and its sections, we employed a factor analysis and cluster analysis, respectively. The results suggest the existence of a relationship between the degree of compliance with the size of mills, and a close relationship between chapters and measurement resources, accountability of management and product realization.
\end{abstract}

\section{Key words}

ISO 9001,quality management, almazara.

$1 \quad$ PhD en Ciencias Veterinarias, Profesora Departamento de Agricultura y Economía Agraria, Universidad de Zaragoza. España

$2 \mathrm{PhD}(\mathrm{c})$ en Ciencias Agrarias y del Medio Ambiente, Docente- investigador grupo USTAGRI Facultad de Administración de Empresas Agropecuarias, Universidad Santo Tomás. Bucaramanga

$3 \mathrm{PhD}$ en Ciencias Agrarias y del Medio Ambiente, Investigador Departamento de Agricultura y Economía Agraria, Universidad de Zaragoza. España

$4 \mathrm{PhD}$ en Ciencias Veterinarias, Director AMB Consultans, Madrid, España 


\section{Introducción}

Los estándares son especificaciones o grupos de especificaciones relativos a las características del producto o de los procesos, que sirven como tipo, modelo, norma, patrón o referencia (Bredahl \& Normile, 2001). En el sector agroalimentario los estándares son, a menudo, legislados o regulados por los gobiernos nacionales, pero, también pueden ser desarrollados e implementados de forma voluntaria en un eslabón de la cadena, ó a través de los diferentes eslabones de la agro-food suply chain (Bredahl \& Normile, 2001; Caswell, 1998; Przyrembel, 2004). Existe evidencia que los estándares voluntarios, como los esquemas de gestión de la calidad y seguridad alimentaria, se han convertido en prácticas empresariales habituales en el sector agroalimentario. Este crecimiento es debido a varias razones, destacándose, i) que los productores y procesadores ven en estos esquemas una forma efectiva de diferenciación; ii) a la respuesta desde la industria sobre la seguridad alimentaria y cambios en los gustos y preferencias de los consumidores; y también, iii) al incremento de los estándares business to business (B2B) solicitados principalmente por los minoristas (Altman, 2006; Bredahl \& Normile, 2001; Fulponi, 2006; Henson \& Reardon, 2005; Maza \& Ramírez, 2005).

La mayoría de los esquemas voluntarios de calidad y seguridad alimentaria están inspirados en la norma ISO 9001, que supone una certificación de tercera parte (Third-Party Certification-TPC) que ofrece sellos de calidad basados en la auditoría de los servicios de certificación (Anders, Souza \& Rouviere, 2007). La información suministrada por una TPC es creíble, ya que la ofrece un tercero que tiene reputación e independencia, tras comprobar la conformidad de las características de un producto o un proceso con los estándares estipulados (Busch, Thiagarajan, Hatanaka, Bain, Flores \& Frahm, 2005; Compés, 2002). En el campo agroalimentario, las principales iniciativas de gestión de la calidad se centran en la seguridad alimentaria, HACCP, ISO 9001, ISO 14000, ISO 22000, BRC, IFS, trazabilidad, región de origen (distintivo de calidad de región de origen), país de origen, comercio justo, buenas prácticas agrícolas, certificación fitosanitaria y certificados ambientales (Busch et al., 2005).

\section{Antecedentes bibliográficos}

En 1987 la Organización Internacional para la Estandarización (ISO) presentó los primeros documentos de la norma ISO 9000 (Withers \& Ebrahimpour, 2000). La norma, que había sido desarrollada para facilitar el comercio armonizaba los términos de calidad, los sistemas y los estándares (Withers \& Ebrahimpour, 2000), consistía en conceptos y directrices generales (ISO 9000), guías de recomendaciones para la gestión de la calidad (ISO 9004) y los tres sistemas de calidad, ISO 9001, 9002 y 9003 (Badía, 2002). En diciembre del año 2000 se aprobó la última edición de la norma, la ISO 9001:2000 que derogaba a las normas internacionales ISO 9001, 
9002 y 9003 de 1994, así como a las equivalentes nacionales que fueran resultado de la correspondiente transposición. En ella se sustituye el concepto de aseguramiento de la calidad por el de gestión de la calidad, éste es más amplio e incorpora la calidad a diferentes aspectos de la gestión de la empresa. La familia de normas ISO 9000 ha quedado estructurada en tres normas básicas: i) la ISO 9000: sistemas de gestión de calidad, fundamentos y vocabulario; ii) la ISO 9001: sistemas de gestión de calidad y requisitos; y iii) la ISO 9004: sistemas de gestión de calidad y directrices para la mejora del desempeño. Posteriormente en 2008 se publicó la nueva norma ISO 9001:2008 que introduce algunos cambios con respecto a la anterior.

"En un comunicado conjunto, tanto la Organización Internacional de Normalización (ISO) como el Foro Internacional de Acreditación (IAF) ha reconocido que la versión de la Norma ISO 9001 de 2008 no incorpora nuevos requisitos sino aclaraciones a los ya existentes, los cuales quedan recogidos mediante la inclusión de nuevas notas y la mejora de la redacción de algunos puntos que, sin duda, redundarán en beneficio de su aplicación por parte de los usuarios. La Norma ISO 9001:2008 mantiene de forma general la filosofía del enfoque a procesos y los ocho principios de gestión de la calidad, que se encuentran recogidos en la Norma ISO 9000:2005, a la vez que es y será genérica y aplicable a cualquier organización independientemente de su actividad, tamaño o su carácter público o privado. Si bien los cambios abarcan prácticamente la totalidad de los apartados de la norma, éstos no suponen un impacto para los sistemas de gestión de la calidad basados en la ISO 9001:2000, ya que fundamentalmente están enfocados a enfatizar o mejorar algunos aspectos" (AENOR, 2009).

La implementación de los estándares ISO 9001 parece ser una tarea casi imposible, porque tiene 150 requisitos específicos (Ferguson, 1996). Se ha identificado que los costes, la cultura empresarial y el tiempo afectan de manera negativa su implementación, sobre todo, en aquellas empresas pequeñas, hasta el punto de requerir la ayuda de consultores externos (Braña, 1995; Ferguson, 1996; Martínez, 1996; Maza \& Ramírez, 2005; Ramírez, 1995; Withers \& Ebrahimpour, 2000). Unido a lo anterior, la falta de personal cualificado sumado al exceso de burocracia, son aspectos que también frenan el proceso de implementación de la norma ISO 9001:2000 (Maza \& Ramírez, 2005; Rubio, 1995).

Enmarcado en este contexto, el presente trabajo tiene fundamentalmente dos objetivos. Por un lado, persigue valorar la relación entre el grado de cumplimiento del sistema de gestión de la calidad ISO 9001 y el tamaño de la empresa en un conjunto de almazaras ubicadas en el sur de España, que eran susceptibles de implantar este modelo de gestión y en las que se realizó previamente un "diagnóstico de calidad". Por otro lado, pretende establecer relaciones entre el grado de cumplimiento de los diversos apartados de la norma en estas agroindustrias en particular. Estos objetivos nos parecen relevantes por cuanto en este momento en el sector agroalimentario no es suficiente competir en el mercado a través de los productos que se ofertan 
en el mismo, sino que se compite fundamentalmente con modelos de gestión. Por ello la profundización en dichos sistemas de gestión es fundamental para valorar las posibilidades de adopción de estos sistemas por parte de las empresas. Asimismo, la mejora de la calidad del aceite de oliva es un factor clave para incrementar la confianza del consumidor final y, por tanto, aumentar el consumo del producto, tanto en la Unión Europea como en terceros países y parece evidente que el objetivo principal de la futura política en el sector del aceite de oliva, será potenciar aún más la calidad del producto.

En el sector del aceite de oliva que aquí nos ocupa existen pocos trabajos que traten sobre la implantación de sistemas de gestión de calidad en los distintos tipos de empresas. Es importante resaltar, que hasta la década de los años noventa, el sector del aceite de oliva en España se basaba en pequeñas almazaras propiedad de los agricultores, y en las industrias de refino y envasado, mucho más concentradas, que compraban este aceite. Hacia el año 2005, existían en España 95 empresas del sector de las grasas y los aceites, certificadas bajo la norma ISO 9001:2000, evidenciándose un enorme incremento a partir del año 1999, ya que, hasta esa fecha sólo existían siete empresas certificadas (MAPA, 2005). Esto podría deberse, a que las cooperativas de segundo grado existentes en el sector, habían introducido criterios empresariales, al concentrar la oferta, mejorar la calidad de los aceites de oliva virgen y colocar su producto en los mercados nacionales e internacionales (Navarro, 2002).

A nivel de explotación agraria, en un reciente trabajo Parra y Calatrava (2005) han caracterizado a los agricultores y a las explotaciones agrarias ecológicas frente a los convencionales en el Sur de España. No podemos decir que la agricultura ecológica sea un sistema de gestión de calidad propiamente dicho, pero sí es cierto que, los productos ecológicos son productos de calidad certificada. Los resultados muestran que los olivareros ecológicos se diferencian de los convencionales en una serie de características de edad, formación y otros. Y, en cuanto a las explotaciones, son en general menos productivas, pero los agricultores tienen una opinión positiva sobre su rentabilidad.

En Alemania, Kane (1995) estudió la aplicación práctica de los requerimientos de ISO 9001 en la compra de aceites y grasas y señaló los pasos necesarios para implementar de forma eficiente un sistema de gestión de calidad. Describió asimismo cómo cumplimentar los requerimientos de ISO 9001 con menos esfuerzo y burocracia.

Referido a las almazaras cooperativas españolas y, más concretamente a las de la región de Castilla-La Mancha acogidas a la denominación de origen Montes de Toledo, el trabajo de Marbán (2005) analiza las posibles estrategias comerciales basadas en esquemas de gestión de la calidad. En él se afirma que el 72,7\% de las almazaras cooperativas estudiadas (22) conocen las normas ISO 9000, aunque sólo el 9,1\% de ellas piensan certificarse. El hecho de que las normas ISO sean bastante 
conocidas se debe, en opinión de Marbán (2005), a que aquellas han empezado a ser consideradas en este sector como parte de una estrategia de diferenciación con el objetivo de penetrar o, incluso, de mantenerse en ciertos mercados, especialmente en los internacionales. No obstante, para Marbán (2005) resulta preocupante que sea tan reducido el número de cooperativas que piensan introducir las normas ISO 9000 , lo cual llevaría a pensar en las dificultades a las que se enfrentan los pequeños y medianos productores a la hora de implantar un sistema de gestión de calidad en su empresa. Asimismo, Marbán (2005) encontró que las estrategias comerciales más valoradas por las grandes superficies son la adopción de las normas ISO, seguidas por la venta de productos amparados en una denominación de origen. Es esperable que las almazaras cooperativas estudiadas incrementen su presencia en tiendas minoristas y grandes superficies, lo que puede provocar un incremento en la adopción de estos sistemas de gestión de la calidad.

También en el sector del aceite de oliva, Camacho, Núñez y Moyano (2005) han desarrollado un modelo de gestión de la calidad basado en las actividades. El modelo denominado ABM, es un instrumento de gestión cuyo objetivo es realizar un control de los costes empresariales en los diferentes niveles organizativos de la empresa. La finalidad, sin embargo, no es gestionar los costes sino las actividades que componen el proceso productivo. Por ello, hace especial hincapié en la distinción entre actividades con y sin valor añadido, al intentar eliminar estas últimas y ejecutar correctamente y con un consumo mínimo de recursos las primeras. El ABM se considera también como una herramienta para evaluar, asegurar y mejorar la calidad de los productos y los servicios ofrecidos por la empresa y, en ese sentido, hace uso de las técnicas de mejora de la calidad, englobándose dentro de la filosofía de la gestión de la calidad total (TQM).

Ghobadian y Gallear (1997) estudiaron las relaciones entre las características del sistema de gestión TQM y el tamaño de la organización, para determinar si el tamaño afecta de forma significativa la adecuación del sistema a la empresa o si existen otras circunstancias que juegan un papel más importante. Los resultados obtenidos parecen indicar que las pequeñas y medianas empresas pueden adoptar fácilmente los principios del sistema TQM; sin embargo, su implementación tiene, en este tipo de empresas, algunos requerimientos específicos. Precisamente, el reconocimiento de estas características diferenciales relativas a distintos aspectos (disponibilidad de recursos, resistencia al cambio, proximidad de los trabajadores y demás) es un factor importante para implementar con éxito el sistema TQM.

Por último, y en relación con las auditorías practicadas en las empresas que han implementado un sistema de calidad de acuerdo a ISO 9000, el trabajo de Liebesman (2002) refleja la utilidad de las auditorías internas para identificar las no conformidades dentro del sistema, y cómo el reconocimiento de las mismas se convierte en oportunidades de mejora. 


\section{Metodología}

La información empleada en el presente estudio proviene de una muestra conformada por 30 almazaras ubicadas en la Comunidad Autónoma Andaluza, en el sur de España, que eran susceptibles de certificación bajo la norma ISO 9001:2000. La recogida de información, que se llevó a cabo durante el último trimestre del año 2004, corresponde a un diagnóstico previo de calidad. Para ello, se emplearon hojas de comprobación donde se describe la situación de las empresas del sector oleícola en aspectos de gestión de la calidad ISO 9001:2000 (ver anexo). Las hojas elaboradas, basadas en la norma UNE-EN ISO 9001:2000 (gestión de la calidad), incluyen un veinte ítems que agrupan los cinco apartados de la norma, es decir, los criterios contractuales: i) sistema de gestión de la calidad, ii) responsabilidad de la dirección, iii) recursos, iv) realización del producto y v) medición.

Las hojas de comprobación han sido utilizadas por auditores (que cumplen las normas UNE-EN ISO 19011:2002) en sus auditorías a las empresas del sector. Estas hojas, permitieron al auditor establecer cinco niveles de conformidad que servirían como sistema de evaluación para determinar la situación de la empresa, conforme a los criterios de gestión de la calidad. Los niveles de conformidad son:

0: Prácticamente no se realiza la acción indicada

1: La acción se realiza parcialmente, en ocasiones puntuales

2: La acción se realiza generalmente, en la mayoría de los casos

3: La acción se realiza sistemáticamente y en casi todas las áreas

4: La acción se realiza siempre y constituye un ejemplo para el sector.

Con las puntuaciones obtenidas en cada uno de los 20 ítems, se estableció el porcentaje de cumplimiento en cada uno de los cinco apartados de la norma, para cada una de las empresas auditadas. Con las 27 variables correspondientes a los ítems de la hoja de comprobación, los porcentajes de cumplimiento obtenidos en cada uno de los apartados de la norma y el tamaño de las empresas, medido por el número de empleados y la facturación anual se realizó, un análisis descriptivo que comprendió fundamentalmente un análisis de frecuencias y medidas de tendencia central e identificación, al mismo tiempo, de datos atípicos. Los datos fueron analizados con el software estadístico SPSS versión 14.0.

\subsection{Clasificación de las empresas y relación con su tamaño}

Con el fin de agrupar las almazaras de acuerdo a su grado de cumplimiento respecto a los cinco apartados de la norma ISO 9001:2000, se llevó a cabo un 
análisis clúster según el método TwoStep (dos fases). A diferencia de los métodos jerárquicos y no jerárquicos, este método de clústering fue seleccionado con el fin de obtener en cierta medida los beneficios que brindan los dos (Hair, Anderson, Tatham \& Black, 1999). La conglomeración en dos fases, es un procedimiento que utiliza un método de clasificación secuencial para obtener una agrupación inicial de los datos (preclasificación) que es posteriormente refinada por un método jerárquico aglomerativo (refinamiento). Como medida de distancia se empleó el Log-verosimilitud y el número de conglomerados se decidió automáticamente. Atendiendo a la medida de distancia empleada y debido a que este método de clústering es bastante robusto, se asumió normalidad en las variables. Las variables empleadas para calcular las distancias fueron los porcentajes de cumplimiento correspondientes a cada uno de los cinco apartados de la norma y el tamaño de las empresas, medido por el número de empleados y la facturación anual en millones de euros. Para evitar inconsistencias en las soluciones finales derivadas de los cambios de escala (porcentajes de cumplimiento y tamaño de las empresas) las variables fueron estandarizadas en puntuaciones Z (Hair et al., 1999).

Establecidos los clúster y asumida la normalidad en todas las variables, se procedió a desarrollar t-tests de comparación de medias entre clúster, se empleó para ello, tanto las siete variables utilizadas en el clústering como las puntuaciones asignadas a cada uno de los 20 ítems de la hoja de comprobación.

\subsection{Relación entre los apartados de la Norma}

Para medir si existe relación en el grado de cumplimiento de los apartados de la norma ISO 9001:2000 en las almazaras estudiadas, se realizó un análisis factorial con los 20 ítems que conforman la hoja de comprobación empleada por los auditores. Por ser el análisis factorial una técnica de interdependencia que reduce información al agrupar en constructos las variables más correlacionadas entre sí (Hair et al., 1999; Uriel y Aldas, 2005), se puede asumir, que los constructos obtenidos, ayudan a determinar la forma en que las almazaras relacionan el cumplimiento de los ítems y apartados de la norma ISO 9001:2000. Para medir el nivel en que las 20 variables empleadas en el análisis factorial se correlacionan entre sí, se empleó el indicador KMO y el test de esfericidad de Bartlett's. El método de extracción de factores utilizado fue el de componentes principales, y dichos factores fueron rotados mediante el método de rotación ortogonal Varimax, de esta forma se pudo entender mejor los factores obtenidos y su correlación con las variables (Hair et al., 1999; Uriel y Aldas, 2005). 


\section{Resultados}

\subsection{Cumplimiento de la norma ISO 9001:2000 y su relación con el tamaño de las almazaras}

El análisis clúster, llevado a cabo, sugiere la existencia de dos conglomerados. Los centroides correspondientes a cada uno de ellos se presentan en la Tabla 1.

Tabla 1. Centroides de los cluster obtenidos.

\begin{tabular}{lccc}
\hline & $\begin{array}{c}\text { Cluster 1 } \\
(\mathrm{n}=15)\end{array}$ & $\begin{array}{c}\text { Cluster 2 } \\
(\mathrm{n}=15)\end{array}$ & Sig. \\
\hline Tamaño de la empresa: Facturación (millones de euros) & 30,79 & 25,61, & No sig. \\
\hline Tamaño de la empresa: Número de empleados & 43,33 & 19,00 & $* *$ \\
\hline Porcentaje de cumplimiento: Sistema de gestiónde la calidad & 0,84 & 0,54 & $* * *$ \\
\hline Porcentaje de cumplimiento: Responsabilidad de la dirección & 0,82 & 0,60 & $* * *$ \\
\hline Porcentaje de cumplimiento: recursos & 0,83 & 0,53 & $* * *$ \\
\hline Porcentaje de cumplimiento: Realización del producto & 0,82 & 0,54 & $* * *$ \\
\hline Porcentaje de cumplimiento: Medición & 0,85 & 0,58 & $* * *$ \\
\hline Nota: $* *$ palue $<0,05, * * * p$ value $<0,001$. No significativo $\alpha=0,05$. & & & \\
\hline
\end{tabular}

Al clúster 1 pertenecen 15 empresas, las más grandes y con mayores porcentajes de cumplimiento de los apartados que componen la hoja de comprobación empleada por los auditores, mientras que las 15 empresas restantes pertenecientes al clúster 2 , por el contrario, se caracterizan por ser más pequeñas y con menores porcentajes de cumplimiento. Las empresas del clúster 1 tienen como promedio 43,33 empleados y 30,79 millones de euros de facturación anual mientras que las del clúster 2, presentan como promedio 19 empleados y 25,61 millones de euros de facturación anual. Aplicado un t-test de comparación de medias se obtuvo, que en los cincos apartados, existen diferencias significativas entre las medias de los porcentajes de cumplimiento de cada clúster, mientras que para el tamaño de la empresa, existen diferencias significativas sólo en el número de empleados y no en el tamaño de la empresa medido por la facturación.

Se puede observar en la Tabla 1, que mientras las empresas pertenecientes al clúster 1 obtuvieron el mayor porcentaje medio de cumplimiento en el apartado correspondiente a medición, las empresas del clúster 2, las más pequeñas, lo hicieron en el apartado responsabilidad de la dirección. Por el contrario, mientras que en las empresas del clúster 1, los apartados correspondientes a responsabilidad de la dirección y realización del producto, son los que poseen los menores porcentajes medios de cumplimiento, en las empresas del clúster 2, el menor porcentaje medio de cumplimiento obedece al apartado recursos.

A nivel más específico, las empresas agrupadas en el clúster 1, presentan mayores niveles de cumplimiento en los 20 ítems de la hoja de comprobación, aunque son los ítems B03, B04 y C10 referidos a que se encuentra documentado el compromiso de la 
alta dirección, se identifican las necesidades y expectativas de las partes interesadas, y son apropiadas las infraestructuras para la consecución de los objetivos, los ítems más valorados. También las almazaras que pertenecen al clúster 2 , coinciden parcialmente al presentar los mayores niveles de cumplimiento en los ítems B03 y B04 (ver Figura 1).

Figura 1. Puntuación media de los ítems que componen la hoja de comprobación

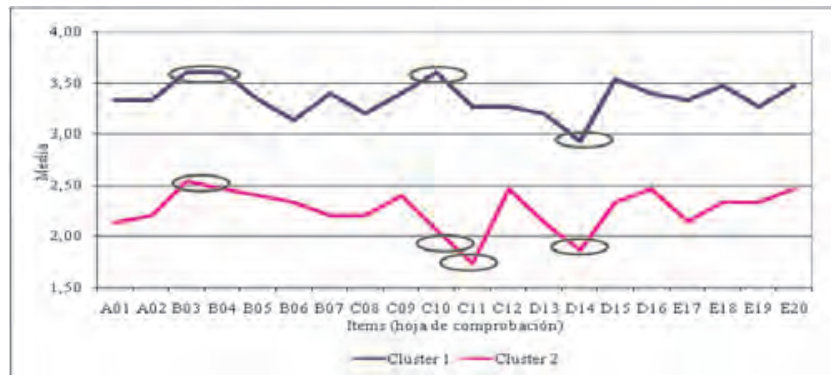

Fuente: elaboración autores

En cuanto a los ítems que presentan menores puntuaciones, destaca el ítem D14 referente a que se encuentran definidos y gestionados los procesos de diseño en el clúster 1 y el C11 referido a que se encuentran implicados los proveedores en la identificación de las necesidades de las compras, en el clúster 2. Es interesante observar, que mientras el ítem $\mathrm{C} 10$ referente a que son apropiadas las infraestructuras para la consecución de objetivos, se encuentra dentro de los más valorados por las empresas que forman el clúster 1, en el clúster 2, por el contrario, se encuentra dentro de los menos valorados. Aplicado el t-test de comparación de medias se obtuvo que existen diferencias significativas ( $p$ value $<0,05$ ) entre las medias de las valoraciones de los dos clúster, de los 20 ítems de la hoja de comprobación.

Respecto a la relación promedio facturación/número de empleados a simple vista pareciera, que el clúster 1 con mayor cumplimiento tiene una desventaja en términos económicos (0,71 millones de euros/empleado) mientras que el clúster 2 , con menor cumplimiento de la norma y con menor número de empleados promedio, presenta una situación mejor (1,35 millones de euros/empleado). Sin embargo, al hacer un t-test de comparación de medias con la relación facturación/número de empleados de cada empresa, se obtuvo que no existen diferencias significativas entre los dos clúster. Esto indica, que la relación facturación/número de empleados como una medida de la productividad laboral parcial, no está asociada de manera significativa $(\alpha=0,05)$ con la adopción de los sistemas de gestión de calidad por parte de las almazaras. 


\subsection{Relación entre los apartados de la Norma}

Los resultados del análisis factorial muestran que los 20 ítems incluidos en las hojas de comprobación para evaluar el grado de conformidad en la implementación del sistema de gestión de la calidad ISO 9001:2000 por parte de las almazaras, se pueden agrupar en 3 factores (ver Tabla 2) que explican el 71,7\% de la información. Estos factores al agrupar variables correlacionadas, se pueden tomar, como las relaciones existentes entre el grado de cumplimiento de los diferentes ítems incluidos en las hojas de comprobación.

El primer factor relaciona fundamentalmente los ítems correspondientes a los capítulos recursos y medición. Por ello puede interpretarse, que en las empresas auditadas, una adecuada disposición de los recursos, entendida como la existencia de adecuadas competencias del personal en función de las necesidades (C09) que demanda cada procedimiento, una buena planificación de los recursos (C08) por parte de la dirección y la existencia de infraestructuras apropiadas para la consecución de los objetivos (C10) marcados, está asociado de manera positiva, con la medición, entendida como la utilización de medidas correctivas y preventivas para la eliminación de problemas (C20), el control de las no conformidades de procesos y productos (E18) y la implantación de procesos de seguimiento y medición (E17), todo esto, unido a la utilización de documentos y registros para la gestión de los procesos (A02), para finalmente obtener un adecuado sistema de gestión de la calidad.

Tabla 2. Resultados del análisis factorial.

\begin{tabular}{|c|c|c|c|c|}
\hline & 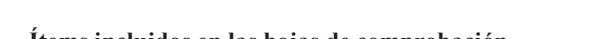 & & ipone & \\
\hline & 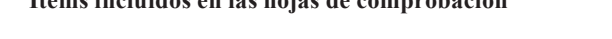 & 1 & 2 & 3 \\
\hline C09: & $\begin{array}{l}\text { Son adecuadas las competencias del personal en función de } \\
\text { las necesidades }\end{array}$ & 0,82 & & \\
\hline E20: & $\begin{array}{l}\text { Se utilizan medidas correctivasy preventivas para eliminar } \\
\text { problemas }\end{array}$ & 0,73 & & \\
\hline C08: & Planifica la dirección los recursos precisos & 0,68 & & \\
\hline $\mathrm{C} 10$ & $\begin{array}{l}\text { Son apropiadas las infraestructuras para la consecución de } \\
\text { los objetivos }\end{array}$ & 0,66 & & \\
\hline E18: & $\begin{array}{l}\text { Se encuentran controladas las no conformidades de } \\
\text { procesos y productos }\end{array}$ & 0,65 & & \\
\hline E17: & $\begin{array}{l}\text { Se encuentran implantados los procesos de seguimiento y } \\
\text { medición }\end{array}$ & 0,64 & & \\
\hline A02: & $\begin{array}{l}\text { Se utilizan documentos y registros para la gestión de los } \\
\text { procesos. }\end{array}$ & 0,63 & & \\
\hline B03: & $\begin{array}{l}\text { Se encuentra documentado el compromiso de la alta } \\
\text { dirección }\end{array}$ & & 0,85 & \\
\hline C11: & $\begin{array}{l}\text { Se encuentran implicados los proveedores en la } \\
\text { identificación de las necesidades de compras }\end{array}$ & & 0,79 & \\
\hline D15: & Se encuentran definidos los procesos de compras & & 0,73 & \\
\hline B04: & $\begin{array}{l}\text { Se idnetifican necesidades expectativas y necesidades de } \\
\text { las partes interesadas }\end{array}$ & & 0,72 & \\
\hline E19: & Se analiza la información referida al sistema de calidad & & 0,58 & \\
\hline
\end{tabular}




\begin{tabular}{|c|c|c|}
\hline B07: & $\begin{array}{l}\text { Revisa la direccción los objetivos de calidad y la eficacia } \\
\text { de lso procesos }\end{array}$ & 0,47 \\
\hline B06: & Se encuentran establecidas las responsabilidades & 0,76 \\
\hline B05: & Se establecen objetivos y metas medibles & 0,68 \\
\hline D14: & $\begin{array}{l}\text { Se encuentran definidos y gestionados los procesos de } \\
\text { diseño }\end{array}$ & 0,67 \\
\hline A01: & $\begin{array}{l}\text { Se aplica el enfoque basados en procesos como práctica de } \\
\text { gestión }\end{array}$ & 0,66 \\
\hline D13: & Se encuentran definidos los procesos relativos a los clientes & 0,63 \\
\hline C12: & $\begin{array}{l}\text { Existe una planificación y control de los recursos } \\
\text { financieros }\end{array}$ & 0,56 \\
\hline
\end{tabular}

Por su parte, de la interpretación del segundo factor se deduce que cuando la alta dirección está comprometida en la identificación de las necesidades de la empresa (B04), se encuentra documentado ese compromiso (B03) y, al mismo tiempo, está inmersa en la revisión de los objetivos de calidad y eficacia de los procesos (B07), se consigue una buena realización del producto, atendidos los procesos de compras (D15) en función de las necesidades de los clientes (D16) y la implicación de los proveedores $(\mathrm{C} 11)$, analizando al mismo tiempo la información referida al sistema de calidad (E19).

El tercer factor incide en la relación encontrada en el anterior entre la responsabilidad de la dirección y la realización del producto en el sentido de que cuando están establecidas las responsabilidades por la dirección de la empresa (B06), y ésta establece objetivos y metas medibles (B05) se consigue una buena realización del producto, encontrándose definidos los procesos relativos a los clientes (D13) y gestionados los procesos de diseño (D14) existiendo al mismo tiempo, una planificación y control de los recursos financieros (C12), y aplicándose un enfoque basado en procesos como práctica de gestión (A01).

\section{Conclusiones}

Teniendo en cuenta el tamaño de las almazaras y su grado de cumplimiento con relación a los apartados que componían la hoja de comprobación empleada por los auditores, se obtuvo la agrupación de las empresas en dos conglomerados. Mientras el primer conglomerado está caracterizado por empresas de mayor tamaño y mayores porcentajes de cumplimiento de la norma ISO 9001:2000, al segundo pertenecen las almazaras más pequeñas y con menores porcentajes de cumplimiento de la norma. Con base en los dos conglomerados obtenidos, se identificó que existe una relación positiva entre el grado de cumplimiento de los apartados de la norma ISO 9000:2000 y el tamaño de las almazaras, medida ésta, por el número de empleados que posee. Esta relación se da en el sentido que mientras mayor sea el tamaño de la empresa, mayor es el nivel de cumplimiento de la norma. Esto podría deberse a que, aunque existe un interés por parte de la alta dirección de los dos grupos de empresas en la implementación de la norma, en las empresas más pequeñas, parece existir cierto 
grado de dificultad a la hora de implementarla. En todos los ítems que conforman la hoja comprobación empleada por los auditores, se hallaron diferencias significativas entre las medias de los dos clúster obtenidos, observándose una mayor valoración por parte de las almazaras que conforman el clúster 1, es decir, las caracterizadas por un mayor tamaño.

El factor 1 pone en evidencia una estrecha relación entre los capítulos recursos y medición, lo que indica que una mayor preocupación de la empresa por una óptima planificación y adecuación de recursos va acompañada de unos mayores controles de medición y seguimiento. Por otro lado, a través de los factores 2 y 3 se constata, que existe una relación estrecha entre los capítulos responsabilidad de la dirección y realización del producto; lo que haría pensar que en las almazaras auditadas, la dirección de la empresa está altamente comprometida con una gestión dirigida hacia los clientes.

\section{Referencias}

AENOR -Asociación Española de Normalización (2009). Comunicado de prensa

Altman, M. (2006). Product policy on the agricultural and food market. Luxembourg: CO CONCEPT

Anders, S., Souza, D.M., \& Rouviere, E. (2007). Objectiveness in the market for Third-Party Certification: does market structure matter? Paper presented at the 105th EAAE Seminar "International Marketing and International Trade of Quality Food Products”, 8-10 March 2007, Bologna, Italy

Badía, A. (2002). Calidad: enfoque ISO 9000. Bilbao, España: Ed. Deusto.

Braña, P. (1995). Calidad e ISO 9000. Qualitas Hodie, 14: 13-14

Bredahl, M.E., \& Normile, M.A. (2001). Thade impacts of voluntary quality standards for livestock products. Paper presented at the Annual Meetings of the International Agricultural Trade Research Consortium, 18-19 January 2001, Auckland, New Zealand.

Busch, L., Thiagarajan, D., Hatanaka, M., Bain, C., Flores, L. G., \& Frahm, M. (2005). The relationship of third-party certification (TPC) to sanitary/ phytosanitary (SPS) measures and the international agri-food trade. United States: United States Agency for International Development, Raises SPS Global Analytical report 9

Camacho, M.M., Núñez, M., Moyano, J. (2005). Modelo de gestión de la calidad basado en las actividades: aplicación específica al sector del aceite de oliva. Alta Dirección, XL(238), 75-83

Caswell, J. (1998). How labeling of safety and process attributes affects markets for food. Agricultural and Resource Economics Review, 27(2), 151-158 
Compés, R. (2002). Atributos de confianza, normas y certificación. Comparación de estándares para hortalizas. Economía Agraria y Recursos Naturales, 2(1), 115 130

Ferguson, W. (1996). Impact of the ISO 9000 series standards on industrial marketing. Industrial Marketing Management, 25, 305-310

Fulponi, L. (2006). Private voluntary standards in the food system: the perspective of major food retailers in OECD countries. Food Policy, 31, 1-13

Ghobadian, A.; Gallear, D. (1997). TQM and organization size. International Journal of Operations \& Production Management, 17(1-2), 121-163

Hair, J., Anderson, R., Tatham, R., \& Black, W. (1999). Análisis multivariante (5 ed.). Madrid, España: Prentice Hall Iberia

Henson, S., \& Reardon, T. (2005). Private agri-food standards: Implications for food policy and the agri-food system. Food Policy, 30, 241-253

Kane, A. (1995). Practical application of the ISO-9001 requirements for the purchasing of oils and fats. Fett Wissenschaft Technologie-Fat Science Technology, 97(1): 29-32

Liebesman, S. (2002). ISO 9000:2000 The challenges and opportunities for internal auditors. Proceedings Annual Reliability and Maintainability Simposium, 28-31 January 2002, Seattle WA, United States

MAPA (2005). La industria alimentaria: empresas certificadas ISO 9000 y 14000. Madrid, España: Ministerio de Medio Ambiente, y Medio Rural y Marino (Antes MAPA)

Marbán, R. (2005). Principales estrategias de gestión de la calidad desarrolladas por las almazaras cooperativas amparadas en la Denominación de Origen Montes de Toledo. CIRIEC- España. Revista de Economía Pública, Social y Cooperativa, 51, 185-209

Martínez, M. (1996). Un sistema de calidad integrador eficaz y generador de sinergias. Qualitas Hodie, 27, 15-16

Maza, M.T.; Ramírez, V. (2005). Study of the main motivations and discouraging factors for the implementation of ISO 9000 standards in spanish agribusiness sector. Journal of International Food \& Agribusiness Marketing, 17(2), 229-243

Navarro, A. (2002). Los mercados internacionales del aceite de oliva y los mercados de graneles de aceite de oliva en España. Distribución y Consumo, 66, 99-117

Parra, C.; Calatrava, J. (2005). Factors related to the adoption of organic farming in Spanish olive orchards. Spanish Journal of Agricultural Research, 3(1), 5-16

Przyrembel, H. (2004). Food labeling legislation in the EU and consumers information. Trends in Food Science \& Technology, 15, 360-365 
Rubio, L.A. (1995). Las industrias alimentarias e ISO 9000. Qualitas Hodie, 14, 20 22

Ramírez, I. (1995). Las ISO 9000 y el sector alimentario. Qualitas Hodie, 14, 26-27

Uriel, E., \& Aldas, J. (2005). Análisis multivariante aplicado. Madrid, España: Thomson Editores Spain

Withers, B. \& Ebrahimpour, M. (2000). Does ISO 9000 certification affect the dimensions of quality used for competitive advantage? European Management Journal, 18(4), 431-443

Anexo 1

\begin{tabular}{|c|c|c|c|c|c|c|}
\hline & \multirow{2}{*}{ Preguntas de comprobación del epígrafe de calidad } & \multicolumn{5}{|c|}{$\begin{array}{l}\text { EVALUACIÓN } \\
\text { AUDITOR }\end{array}$} \\
\hline & & $\mathbf{0}$ & 1 & 2 & 3 & 4 \\
\hline A1 & Se aplica el enfoque basado en procesos como práctica de gestión & & & & & \\
\hline A2 & Se utilizan documentos y registros opara la gestión de procesos & & & & & \\
\hline A & TOTAL SISTEMA DE GESTIÓN DE LA CALIDAD & & & & & \\
\hline B3 & Se encuentra documentado el compromiso de la alta dirección & & & & & \\
\hline B4 & Se identifican necesidades, expectativas y necesidades de las partes interesadas & & & & & \\
\hline B5 & Se establecen objetivos y metas medibles & & & & & \\
\hline B6 & Se encuentran establecidas las responsabilidades & & & & & \\
\hline B7 & Revisa la dirección los objetivos de calidad y la eficacia de los procesos & & & & & \\
\hline $\mathrm{B}$ & TOTAL RESPONSABILIDAD DE LA DIRECCIÓN & & & & & \\
\hline $\mathrm{C} 8$ & Planifica la dirección de los recursos precisos & & & & & \\
\hline C9 & Son adecuadas las competencias del personal en función de las necesidades & & & & & \\
\hline $\mathrm{C} 10$ & Son apropiadas las infraestructuras para la consecución de los objetivos & & & & & \\
\hline C11 & Se encuentran implicados los proveedores en la identificación de las encesidades de compras & & & & & \\
\hline $\mathrm{C} 12$ & Existe una planificación y control de los recursos financieros & & & & & \\
\hline C & TOTAL RECURSOS & & & & & \\
\hline D13 & Se encuentran definidos los procesos relativos a los clientes & & & & & \\
\hline D14 & Se encuentran definidos y gestionados los procesos de diseño & & & & & \\
\hline D15 & Se encuentran definidos los procesos de compras & & & & & \\
\hline D16 & Se encuetnran asegurados las consideraciones de las necesidades de los clientes & & & & & \\
\hline D & TOTAL REALIZACIÓN DEL PRODUCTO & & & & & \\
\hline E17 & Se encuentran implantados los procesos de seguimiento y medición & & & & & \\
\hline E18 & Se encuentran controladas las no conformidades de procesos y productos & & & & & \\
\hline E19 & Se analiza la información referida al sistema de calidad & & & & & \\
\hline E20 & Se utilizan medidas correctivas y preventivas para eliminar problemas & & & & & \\
\hline $\mathbf{E}$ & TOTAL MEDICIÓN & & & & & \\
\hline $\mathbf{F}$ & TOTAL CALIDAD $(\mathbf{A}+\mathrm{B}+\mathrm{C}+\mathrm{D}+\mathrm{E})$ & & & & & \\
\hline
\end{tabular}

Niveles de valoración: $0=$ Prácticamente no se realiza: $1=$ Se realiza parcialmente (en ocasiones puntutales); $2=$ Se realiza generalmente (en la mayoria de los casos):; $3=$ Se realiza sistemáticamente y en casi todas las áreas; $4=$ Se realiza siempre u de forma total 